\title{
Erratum to: The distribution of inelastic dark matter in the Sun
}

\author{
Mattias Blennow $^{1,2, a}$, Stefan Clementz ${ }^{1, b}$, Juan Herrero-Garcia ${ }^{3, c}$ \\ ${ }^{1}$ Department of Physics, School of Engineering Sciences, KTH Royal Institute of Technology, AlbaNova University Center, \\ 10691 Stockholm, Sweden \\ ${ }^{2}$ Instituto de Física Teórica UAM/CSIC, Calle Nicolás Cabrera 13-15, Cantoblanco, 28049 Madrid, Spain \\ ${ }^{3}$ ARC Center of Excellence for Particle Physics at the Terascale (CoEPP), University of Adelaide, Adelaide, SA 5005, Australia
}

Received: 9 April 2019/ Accepted: 22 April 2019 / Published online: 14 May 2019

(C) The Author(s) 2019

\section{Erratum to: Eur. Phys. J. C (2018) 78:386} https://doi.org/10.1140/epjc/s10052-018-5863-4

The annihilation rates in Fig. 9 of the original article were incorrectly calculated, which led to erroneous conclusions. The figure is calculated as an upper bound on the annihilation rate. It is therefore possible to state that if the upper bound on the annihilation rate falls below the solar capture rate, there is definitely not equilibrium between solar capture and annihilations. This important assumption is used when constraining the DM-nucleon cross section via the non-observation of high energy neutrinos from DM annihilation in the Sun. The erroneous conclusion based on Fig. 9 of the paper is that there is almost never equilibrium between the solar capture rate and annihilation.

The correct annihilation rates are presented in Fig. 9. As can be seen, throughout most of the parameter space analysed the upper bound on the annihilation rate is greater than the capture rate, the exception being for $m_{\chi}=$ $500 \mathrm{GeV}$ and $\delta \gtrsim 150$. Given these results, it is impossible to state whether equilibrium between capture and annihilations has taken place except for in the aforementioned range in parameters (where equilibrium has not been reached).

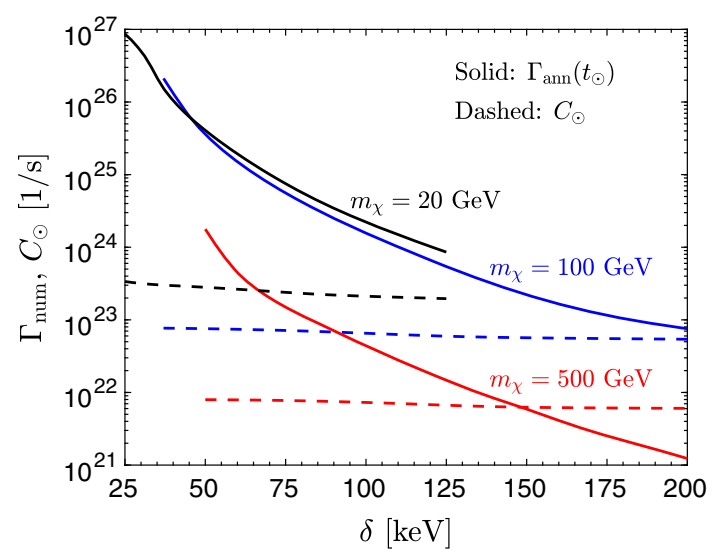

Fig. 9 A comparison between solar capture rates (dashed lines) and the upper bound on the annihilation (solid lines). Results are shown for the dark matter masses: $m_{\chi}=20 \mathrm{GeV}$ (black), $m_{\chi}=100 \mathrm{GeV}$ (blue) and $m_{\chi}=500 \mathrm{GeV}$ (red)

Open Access This article is distributed under the terms of the Creative Commons Attribution 4.0 International License (http://creativecomm ons.org/licenses/by/4.0/), which permits unrestricted use, distribution, and reproduction in any medium, provided you give appropriate credit to the original author(s) and the source, provide a link to the Creative Commons license, and indicate if changes were made.

Funded by SCOAP ${ }^{3}$.

The original article can be found online at https://doi.org/10.1140/ epjc/s10052-018-5863-4.

\footnotetext{
a e-mail: m.blennow@csic.es

be-mail: scl@kth.se

c e-mail: jherrero@sissa.it
} 